\title{
Sigmoid volvulus: Treatment options and volvulus recurrence
}

\author{
Sabri Selcuk Atamanalp, Rifat Peksoz \\ Department of General Surgery, Faculty of Medicine, Ataturk University, Erzurum, Turkey
}

\begin{abstract}
Sigmoid Volvulus (SV) is principally treated with non-operative decompression, whereas it may require surgical management in some situations. Depending upon the treatment method, SV recurs in about one fourth of the cases, which doubles the mortality and morbidity rates. In this paper, we discuss the management and related recurrence of SV in the light of the largest single-center SV series over the world.
\end{abstract}

\section{Highlights}

Endoscopic decompression is the primary treatment option in uncomplicated or non-gangrenous patients with sigmoid volvulus.

- In patients treated with decompression alone, volvulus recurrence is an expected outcome, which doubles mortality and morbidity rates.

- In selected patients, sigmoid colectomy or percutaneous endoscopic colopexy may prevent or reduce the recurrence.

Correspondence: Sabri Selcuk Atamanalp, Department of General Surgery, Faculty of Medicine, Ataturk University, 25040 Erzurum, Turkey.

Tel.: +90.442.3447560, Fax: +90.442 .3446528 .

E-mail: ssa@atauni.edu.tr

Key words: Sigmoid volvulus; treatment; recurrence.

Conflict of interest: The authors have no conflict of interest to declare.

Availability of data and materials: Data are available upon request from the corresponding aurhor.

Ethics approval and consent to participate: This study was approved by institutional review board (Ethics Committee of Ataturk University Faculty of Medicine, 220/2020).

Informed consent: Informed consent was obtained from all participants.

Received for publication: 22 November 2021.

Revision received: 28 November 2021.

Accepted for publication: 28 November 2021.

This work is licensed under a Creative Commons Attribution 4.0 License (by-nc 4.0).

(C) Copyright: the Author(s), 2021

Licensee PAGEPress, Italy

Emergency Care Journal 2021; 17:10294

doi:10.4081/ecj.2021.10294

\section{Introduction}

Sigmoid Volvulus (SV) is a rare disease, in which the sigmoid colon twists around its own mesentery. ${ }^{1}$ Non-operative decompression is tried in uncomplicated or non-gangrenous cases, while patients with above-mentioned adverse situations or those with unsuccessful endoscopic decompression are treated with emergency surgery. ${ }^{2,3}$ Volvulus Recurrence (VR) is an expected result in some SV cases and it worsens the prognosis. ${ }^{4}$ We discuss herein our experiment on the treatment of SV as well as the prevention of the VR by evaluating the largest single-center SV series over the world, ${ }^{5}$ a 1,040-patient experience with SV over a 55 -year period between June 1966 and July 2021.

\section{Materials and Methods}

The clinical records of 612 patients treated until June 1986 were reviewed retrospectively, whereas 428 cases treated thereafter were evaluated prospectively. For each patient, treatment method, success status, mortality, morbidity, and recurrence were noted.

\section{Results}

Non-operative decompression (barium enema in 13, rigid endoscopy in 351, flexible endoscopy in 397) was tried in 761 patients $(73.2 \%)$ with $83.0 \%$ of success, $0.7 \%$ of mortality, $2.2 \%$ of morbidity, and $5.6 \%$ early recurrence rates. Of the patients, 478 $(46.0 \%)$ were treated by emergency surgery with $16.9 \%$ of mortality, $34.1 \%$ of morbidity, and $0.6 \%$ of early recurrence rates. In patients with successful non-operative decompression, elective surgery (open in 95, laparoscopic in 19) was tried in 114 patients $(19.2 \%)$ with $0.0 \%$ of mortality, $11.4 \%$ of morbidity, and $0.0 \%$ of recurrence rates.

\section{Discussion}

In the treatment of uncomplicated and non-gangrenous patients with SV, the first step is the confirmation of the disease by demonstrating a spiral twist of the sigmoid lumen in endoscopic examination (Figure 1a). Following this, contrast agent enema or preferably endoscopic decompression is the optimal option, the latter consisting of gentle insertion of the rigid or preferable flexible endoscope with minimal air insufflation and gentle rotation. Nonoperative procedures may be applied with a $48-100 \%$ success rate, ${ }^{6-8}$ which is compatible with our results. Complicated or gangrenous patients, or patients with unsuccessful endoscopic decompression, are treated by emergency surgery. In non-gangrenous patients, decompression alone or together with a volvulus-reducing procedure including sigmoid resection, sigmoidopexy, 

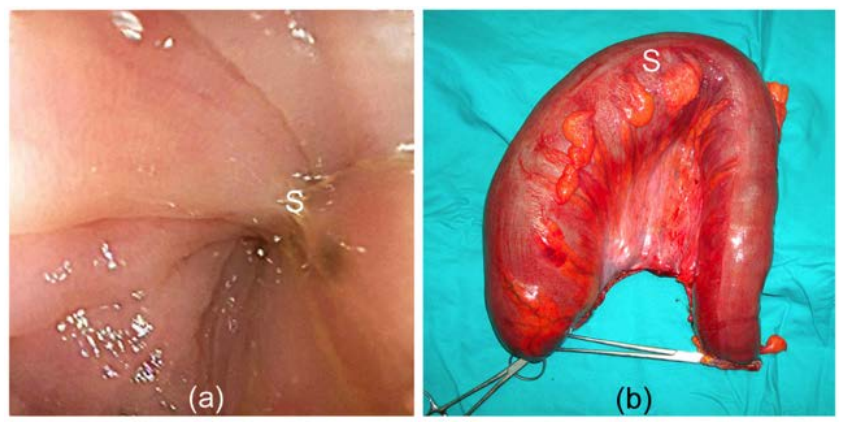

Figure 1. (a) Endoscopic appearance (S, spiral sphincter-like twisted viable sigmoid lumen), and (b) postoperative appearance (S, resected non-gangrenous sigmoid colon).

mesopexy, or mesoplasty is used. In gangrenous patients, sigmoid resection with primary anastomosis or stoma is preferred. ${ }^{3}$ Our results related to the surgical treatment are compatible with the literature findings. On the other hand, in patients with successful non-operative decompression, to prevent a VR, elective sigmoid resection is suggested in nonelderly and well-conditioned patients, ${ }^{3,9}$ which procedure did any good in our series. As an alternative, Percutaneous Endoscopic Colopexy (PEC) may be used in elderly or bad-conditioned patients for the same purposes. ${ }^{3,10}$

VR occurs in a mean $25 \%$ of the patients with SV (range: 0 $55 \%$ ), particularly in patients treated with endoscopic or surgical decompression alone. To prevent the VR, elective sigmoid colectomy with primary anastomosis, preferably by using laparoscopy, is recommended in selected patients. ${ }^{9}$ However, the selection criteria are not objectively identified in literature. ${ }^{6,7,9,10}$ In our opinion and experience, elective surgery may be suggested in patients under 70-75 years old and with American Society of Anesthesiologists (ASA) classes 1-3, whose estimated operative mortality rates are less than $4.3 \%$. On the other hand, PEC, preferably as an elective procedure, may be tried in patients over 75 years old or with ASA class $\geq 4$ patients, whose estimated operative mortality rates are higher than $7.8 \%$. Regarding the technical details of elective sigmoid resection, which is another unidentified subject, $6,7,9$ in our opinion and experience, a maximum length of the sigmoid colon, which allows a tension-free anastomosis without requiring a left colon mobilization, must be resected to prevent the VR (Figure 1b).

\section{Conclusions}

Although the emergency treatment of SV is important, the avoidance of VR, an expected result of SV, is as important as its treatment. For this reason, elective sigmoid colectomy or elective PEC must be considered in selected patients.

\section{References}

1. Lai SH, Vogel JD. Diagnosis and management of colonic volvulus. Dis Colon Rectum 2021;64:375-8.

2. Atamanalp SS. Expert commentary on the diagnosis and management of colonic volvulus. Dis Colon Rectum 2021;64:3789.

3. Atamanalp SS. Sigmoid volvulus: An update for Atamanalp classification. Pak J Med Sci 2020;36:1137-9.

4. Atamanalp SS, Atamanalp RS. Sigmoid volvulus: avoiding recurrence. Tech Coloproctol 2019;23:405-6.

5. Atamanalp SS. Sigmoid volvulus: the first one thousand-case single-center series in the world. Eur J Trauma Emerg Surg 2019;45:175-6.

6. Miller AS, Boyce K, Box B, et al. The Association of Coloproctology of Great Britain and Ireland consensus guidelines in emergency colorectal surgery. Colorectal Dis 2021;23:476-547.

7. Alavi K, Poylin V, Davids JF, et al. The American Society of Colon and Rectal Surgeons clinical practice guidelines for the management of colonic volvulus and acute colonic pseudoobstruction. Dis Colon Rectum 2021;64:1046-57.

8. Atamanalp SS. Endoscopic decompression of sigmoid volvulus: Review of 748 patients. J Laparoendosc Adv Surg Tech 2021; https://doi.org/10.1089/lap.2021.0613

9. Seow-En I, Chang SC, Ke TW, et al. Uncomplicated sigmoid volvulus is ideal for laparoscopic sigmoidectomy with transrectal natural orifice specimen extraction. Dis Colo Rectum 2021;64:e90-3.

10. Jackson S, Hamed MO, Shabbir J. Management of sigmoid volvulus using percutaneous endoscopic colostomy. Ann R Coll Surg Engl 2020;102:654-62. 